\begin{tabular}{|c|c|c|c|c|}
\hline Revista Praxis & ISSN: $1657-4915$ & Vol. 12 & 135 - 144 & Enero - Diciembre de 2016 \\
\hline \multicolumn{5}{|c|}{ DOI: http://dx.doi.org/10.21676/23897856.1855 } \\
\hline
\end{tabular}

\title{
PARA QUÉ UN APRENDIZAJE CONTEXTUALIZADO Y COHERENTE EN LA ESCUELA
}

\author{
WHAT A CONTEXTUALIZED LEARNING AND COHERENT IN SCHOOL
}

\author{
Carlos Andrés Ortega-García ${ }^{1}$
}

\begin{abstract}
RESUMEN
Se presentan los resultados correspondientes a una investigación sobre el ejercicio docente en la Institución Educativa 20 de Julio-Escuela Ignacio Renjifo, Cali, 2003-2004. El objeto de estudio fue el grupo Primero (grupo A, jornada de la tarde), compuesto por niños que oscilaron entre los 5 y los 8 años de edad. Después de un trabajo de observación frente a las dinámicas de comprensión de la información, de las normas y de sus relaciones interpersonales, se pudo tener un punto de inicio: comprender los mecanismos por medio de los cuales los estudiantes aprenden a leer y a escribir. Meta que estaba relacionada con propuestas normativas ajenas a los insumos culturales propios de los estudiantes. La metodología usada para analizar el proceso de educación se centró particularmente en la observación de las estrategias comunicativas implementadas dentro y fuera del aula. Estos elementos que fueron guiando la pedagogía implementada en el desarrollo del curso, bajo una trilogía vital que entrelazó: Contexto, individuo y academia.
\end{abstract}

Palabras clave: Pedagogía, espacio escolar y comunidad educativa.

\begin{abstract}
The results of a research about the teaching practice at the Institución Educativa 20 de Julio-Escuela Ignacio Renjifo, Cali, 20032004 are presented. The subject of study was the first grade (group A, afternoon shift), which is composed by children with an age range between 5 and 8 years old. After the observation of the dynamics of the understanding of information, norms and interpersonal relationships, it was possible to have a starting point: understand the mechanism through the students learn to read and write. This goal was related with legislative proposals which were foreign to the own cultural supplies of the students. The methodological view to analyze the process of education focused particularly on the observation of communicative strategies implemented inside and outside the classroom. Guiding elements were implemented in the pedagogy course development, under a vital laced trilogy: context, individual and academia.
\end{abstract}

Keywords: Education, school space and education community.

Fecha de recepción: Julio 30 de 2015 / Fecha de aceptación: Agosto 10 de 2016

Tipología: Artículo de Reflexión

Para citar el artículo: Ortega, G. C. (2016). Para qué un aprendizaje contextualizado y coherente en la escuela. Praxis. Vol. 12, 135 - 144

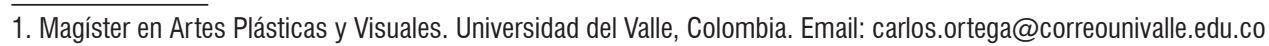




\section{INTRODUCCIÓN}

Ц a experiencia permitió identificar graves uproblemas en el proceso formativo, muchas de estas observadas por fuera del salón de clase: Los planes de aula anteriores se construyeron sin tener en cuenta al estudiantado, infraestructura deteriorada, relaciones interpersonales precarias, apatía de muchos niños con respecto al estudio y responsabilidad ausente de padres de familia en el proceso educativo de sus hijos e hijas. Estos factores adversos fueron tenidos en cuenta para repensar las dinámicas escolares, asumiendo desde el análisis la vinculación de la comunidad académica en el ejercicio de enseñanza-aprendizaje. El presente documento registra un proceso de exploración pedagógica que se construyó con la comunidad misma, fue sin duda, una experiencia formativa ajena a la que habían tenido hasta ese momento: estudiantes, padres de familia y docente.

Este proceso educativo le brindó participación activa a los estudiantes y padres de familia, reconociendo que el ejercicio escolar es una apuesta que inmiscuye a la comunidad en general. Los lineamientos de enseñanza-aprendizaje alejaron la aprehensión de un conocimiento específico y reglamentado, el cual se impone a través de una visión formativa de obediencia. Por el contrario, se centró en una dinámica más flexible, más cariñosa y más rigurosa frente a la búsqueda de alternativas para gozarse el ambiente académico. El documento se estructura a partir de una investigación que triangula individuo, contexto y escuela. Asumiendo que la búsqueda de mejores alternativas pedagógicas se debe centrar en el análisis del ejercicio docente y en la comprensión del saber por parte del estudiantado.

La metodología puesta en marcha requirió de la participación de los educandos en escenarios extra escolares, lo que indicó que el compromiso de aprender no sólo es con la academia, también lo es con la ciudad, con la comunidad que acoge aquellos pequeños y les permite convertirse en mejores seres humanos al pasar por una institución educativa.

\section{El ejercicio de comprensión de la labor docente en la escuela}

La educación es una alternativa social en Colombia, enmarcada por supuesto dentro de unos parámetros de privilegios económicos que estratifican a los espacios escolares. Más allá de esta problemática de jerarquización de escuelas, en la que unos escenarios están nutridos con toda la tecnología y ambientación necesaria para un escenario de aprendizaje ideal, otros funcionan en una precariedad absoluta. La escuela Ignacio Rengifo pertenece a este segundo grupo de instituciones caleñas, en la que los inconvenientes de la infraestructura y los recursos pedagógicos, están acompañados por otra serie de inconvenientes situados dentro de aspectos económicos y culturales de la comunidad estudiantil.

El docente-investigador buscó hacer caso omiso de estas falencias para desarrollar con sus estudiantes un proceso de enseñanza-aprendizaje ajustado a los requerimientos señalados desde la dirección, teniendo a disposición un salón de 20 metros cuadrados para 34 estudiantes, un tablero, una escritorio y 34 asientos. Pasados 8 días, la situación de goce y disfrute de las actividades escolares era sólo una ilusión del educador. De alguna forma se comparte la idea de que las condiciones complejas dificultan la labor del profesor. “[...] no existe un modelo pedagógico único, omnipotente, capaz de solucionar todos los problemas de aprendizaje que tienen los estudiantes [...]" (Ortiz, 2011, p.124) Sin embargo, el ejercicio se empeña en encontrar dinámicas que contribuyan a tener un mejor ambiente escolar. Es así como se inicia analizando lo que se tiene: Los infantes evidenciaban una dinámica somnolienta que frenaba el despertar de la conciencia educativa, muchos de ellos llegaban sin almorzar y sin bañarse. La concentración en los ejercicios era más una obligación que un disfrute, no se conseguía que se dedicaran a la misma actividad por más de 10 minutos.

Fue claro que no existía ningún interés del estudiantado para desarrollar el programa de trabajo, es muy complejo aceptar que todo lo 
planeado antes de iniciar clase es un rotundo error, se optó entonces por conocer quiénes eran aquellas personas sentadas enfrente del escritorio del profesor. Niños y niñas sin la menor idea de lo que significa educación escolar: ¿Para qué asistir a la escuela?, ¿por qué creen que están acá?, ¿qué metas tienen al finalizar este proceso educativo que dura un poco más de 10 años? Un $90 \%$ de ellos reconoció la escuela como aburridora y señalaron otras opciones como divertidas; la televisión, la fiesta, el fútbol y las peleas. Definitivamente los medios de comunicación han construido referentes que se mueven alrededor de los jugadores de fútbol, los pandilleros, los mafiosos o los protagonistas de los reality, personajes bien conocidos por todos.

Indiscutiblemente la condición socio-cultural de los pequeños influye directamente en este anhelo por visualizarse a futuro. El tema de la escuela pública ha sido explorado de forma continua y ha determinado una preocupación constante por el desconocimiento o por la despreocupación de los docentes para conocer las necesidades de sus estudiantes, de igual manera, el vínculo entre escuela y familia es cada vez más limitado. Con este planteamiento, se realizó una reunión informativa, en ésta se expuso a los estudiantes y a sus acudientes, que la responsabilidad de la educación correspondía a un ejercicio compartido, en el cual la Comunidad Académica (directivos, docentes, estudiantes y padres de familia) deben estar en constante diálogo para afinar cuantas veces sea necesario la propuesta escolar.

Se debe decir que la escuela es un escenario de interacción social donde las relaciones humanas se suponen favorables a los sujetos inmiscuidos en aquel espacio, el término favorable está unido a buenos parámetros de convivencia, o a la solución equitativa de los conflictos. El aula de clase se concibe entonces como un campo dialógico, de interacción y de participación social, que le posibilita a los agentes allí inmersos exponer sus vivencias en relación a sus alegrías o frustraciones, a través de un lenguaje polifónico, "la polifonía se refiere a la intervención de discursos o fragmentos discursivos, de géneros discursivos del pasado, en un discurso presente, del aquíahora" (Beltrán, 2005, p.62). El escenario escolar evidencia como "La pedagogía no es solo enseñar al otro un acervo de saberes, sino conseguir que una persona con otra puedan decir coejecutivamente 'estamos creciendo en la inteligencia'" (Murcia, 1991, p.26). La intención educativa se supone que potencia entonces la búsqueda del conocimiento de sí mismo, permitiéndole al sujeto emprender un arduo camino hacia su desarrollo personal, profesional y ciudadano, pero el tiempo es limitado y las reuniones que se supone son puntos claves en este diálogo, se reducen a la narración de resultados, además de caracterizarse por una inasistencia amplia.

Evidentemente el proceso de crecimiento personal desborda las paredes de la escuela, y debe estar sustentado en un ambiente de credibilidad o aceptación (Zambrano, 2001), sin embargo, la obligatoriedad y el castigo siguen siendo recursos para imponer estos procesos de formación. El ambiente académico se concibe como un espacio de interacción social, en el cual se establecen relaciones encaminadas a fortalecer el proceso formativo de los sujetos que la estructuran.

A juzgar por las investigaciones y decisiones que toman los gobiernos, en todo el mundo se espera que la escuela resuelva los problemas que genera el saber como producto y los saberes posibles que se involucran en una sociedad, lengua, tradiciones, creencias, ciencia, técnica, arte y religión”. (Cano, 2006, p. 33)

El espacio escolar de igual manera es el escenario que le permite al hombre iniciar su reconocimiento más allá del círculo familiar, posibilitándole a este comprender lo que es como individuo con respecto al otro, asignándole sentido a su vida.

El mismo concepto de espacio escolar y los recursos a su disposición, ahora, nos obligan hablar de escuelas orientadas a polos de excelencia que optimicen con gestión de calidad los recursos materiales y humanos en la zona donde la escuela está ubicada. (Vásquez, 2009, p.18)

Sin embargo, en la indagación, los insumos o los recursos docentes utilizados, son ajenos al contexto y a los individuos, lo que hace imposible 
hablar de coherencia pedagógica con respecto a lo enseñado.

Las dinámicas de interacción que se propician en el ambiente escolar, motivan una serie de cuestionamientos: ¿bajo qué perspectivas se forma el educador que integra la escuela? Indiscutiblemente, esta encarna una responsabilidad muy alta con la sociedad, esto indica:

que los programas de formación deben partir de la experiencia diaria docente, es decir de las características de cada escuela y de sus maestros, alumnos y comunidades donde ella se encuentra y que los maestros deben participar activamente en su concepción y en su desarrollo”. (Cárdenas, 2000, p. 63)

Lo que determina que el proceso educativo debe responder a un análisis contextualizado, antes que a un saber planteado en fórmulas foráneas que desconocen las distintas problemáticas sociales.

Mirar el contexto para el cual se está trabajando es una labor muy importante, pero concientizar al profesor de este ejercicio, es vital para ofrecer mejores perspectivas educativas a los educandos. Otra pregunta sería: ¿Cómo se puede despertar la conciencia de comprender la escuela más allá del aula de clase?

la escuela es universal a pesar de todo. O sea, en la calle; cuando hablas de estas cosas en la verdulería, con la gente, la escuela está en ese momento en la verdulería. Esto significa que el proceso escolar no solo tiene lugar en la escuela, sino que empieza en cuanto un ser humano habla con otro de esas cosas". (Beuys, 1995, p.33)

Si el docente lo aplica, le será más fácil explicarle al niño que su proceso de aprendizaje continúa por fuera del salón. La escuela encarna un proceso que se debe hacer evidente y que está implícito cuando se comparten ideas o cuando se aprende en relación a un proceso de indagación contextualizado o cuando se puede a partir de lo particular evidenciar la totalidad.

La función de la escuela se supone participativa y propositiva, aunque de forma más concreta, "se trata de permitir a todos los niños que aprendan a leer, escribir y contar, de dotarse de los programas escolares necesarios para su desarrollo personal, su integración social y su éxito profesional” (Meirieu, 2004, p. 21). Incluso ser constructivista en términos del francés Jean Piaget, aunque para el caso de la investigación, nunca fue solo asimilar el saber desde el escenario escolar, la preocupación fuerte se dio con respecto a la transformación activa de las relaciones humanas, a partir de la concientización del estudiante para entenderse como ciudadano o como proyecto de él. Por tanto, es a través de la educación como los sujetos acceden a la posibilidad de comprender que su proceso formativo está en relación consigo mismo, y con su entorno. Es importante determinar que la comprensión del contexto, debe realizarse a partir de un método, de un conocimiento o si se quiere de una visión,

...el aprendizaje sólo podrá surgir si desde afuera un ser, una institución o una herramienta, vienen a proporcionarme los elementos sin los cuales estaría sordo, ciego y mudo para siempre. Ya que aprender es comprender, es decir, recoger para mi unas porciones de este mundo exterior, integrarlas en mi universo y construir así sistemas de representación más perfectos, que ofrezcan cada vez más posibilidades de acción sobre este mundo”. (Meirieu, 2002, p. 41)

El espacio escolar genera entonces de manera activa una reflexión permanente del sujeto con su historia y es a partir de esta acción que este desarrolla una actitud coherente con su pasado, no desde una estadística cronológica, sino desde la capacidad de explicarse como ciudadano.

Comprenderse como ciudadano o en proyecto de serlo es un aval que irá fortaleciendo el proceso educativo, en el cual la figura del profesor es vital. Se precisa que el análisis del ejercicio docente debe contribuir al mejoramiento de escenarios más acordes y a la implementación de dinámicas pedagógicas contextualizadas. La investigación se centró en esta observación determinando que las alternativas de enseñanza - aprendizaje no sólo corresponden a una labor del educador en solitario, todo lo contrario, éste debe estar en 
capacidad de liderar la participación de la comunidad inmiscuida. Siempre desarrollándose una reflexión basada en el acto educativo, específicamente en lo correspondiente a la interacción entre el sujeto que se educa, su contexto y las distintas dinámicas del saber que se imparten en la escuela.

Para el inicio del proceso se planteó la intención de propiciar desde el análisis una metodología consciente de la condición del deseo de saber de cada educando, independiente de su edad o de su nivel académico. Es inevitable asumir que dentro de todo proceso educativo existe siempre la mejor intención de parte y parte (profesor y/o estudiante) para participar en la transferencia del conocimiento. Sin embargo, la consulta entre los profesores determinó que en general los proyectos de aula se trazaron sin la previa participación del estudiantado. Dichos documentos fueron realizados bajo la mirada de un docente confiado en que su proceder sólo debía estar acorde con un saber académico, antes que con un saber social o humano.

La figura del profesor, se dimensiona desde la autoridad, desconociendo el acervo cultural que ha nutrido a los estudiantes en su desarrollo histórico, son ellos mismos los que le otorgan dicha condición a su docente, aunque es claro que éste tiene para garantizar el orden a través de las normas, las cuales corresponden a la manera correcta como se supone que el individuo se debe comportar, y se asume como un determinante de la formación misma, no sólo en el ámbito académico. "Al igual que la mente, la cultura funciona como un ordenador: genera una manera común de apreciar y conducirse ante la realidad. Esa manera es la norma, y a ella se tiene como ideal o modelo" (Iglesias, 2007, p. 52). La educación básica para Iglesias, se construye a partir de parámetros normativos orientados hacia la formación de una personalidad guiada a partir del cumplimiento de los contenidos programáticos y en la obediencia de las bases morales establecidas al interior del aula.

Este proyecto alteró los parámetros normativos institucionales y contempló nuevas intensiones educativas, sugiriendo dinámicas más amigables de formación o mejor de educación, que se centraron en despertar en los estudiantes el deseo de aprender. Lo anterior indica, que este deseo es la esencia educativa del proceso e inicia en el reconocimiento del ser humano y su entorno.

El proyecto fue revelando las condiciones contradictorias que enfrentan a nivel colectivo los estudiantes en estudio, es claro que las falencias con que se educan los infantes son indiscutibles. El inconveniente es semejante en otros contextos, en una investigación realizada durante la década de 1990, en Chile, se identificaron características similares, los resultados fueron desfavorables:

Existe suficiente evidencia entonces, para concluir que las escuelas básicas que atienden a los sectores sociales más pobres no aseguran todavía los conocimientos iniciales $\mathrm{f} \mathrm{u} \mathrm{n} \mathrm{d} \mathrm{a} \mathrm{-}$ mentales (lecto-escritura y matemáticas) que les permitan acceder a los restantes saberes, limitando de esa forma gran parte de su posible aprendizaje futuro. (Concha, 2006, p. 89)

Encontrar una alternativa de aprendizaje adecuada fue un proceso dispendioso, siempre se buscó ofrecer un ambiente coherente con el contexto, respetuoso del entramado cultural de los estudiantes y ajustado a las intenciones escolares (enseñar a leer y a escribir). Se inició con las relaciones interpersonales, como grupo se establecieron deberes y derechos, siendo el deporte la primera opción para establecer acuerdos de interacción, era necesario ejemplificar desde estas prácticas lo que posteriormente se estructuraría en el salón de clase. Siempre se visualizaron líderes que determinaron la manera de jugar, sin embargo, en el salón, el grupo estaba a la expectativa de lo sugerido por el profesor.

Por otro lado, los padres de familia se mostraron en un principio confundidos con el desplazamiento de intereses escolares académicos, ellos confiaban en la trascendencia de iniciar el aprendizaje de la lectura y la escritura, al iniciar el año lectivo; según argumentos expuestos por muchos de ellos en la primera reunión. Se les expuso entonces que más allá de un saber académico, estaba la idea de colaborar en la constitución de un buen ser humano, por tal motivo, se realizaría 
una labor constante para incentivar el respeto con sigo mismo y con sus compañeros.

En una segunda fase de trabajo, estaba el comportamiento como grupo, para su aplicación se propiciaron salidas de campo: experiencias registradas fuera del aula, luego fuera de la institución y, por último, fuera del barrio. Cada uno de los recorridos arrojó características satisfactorias, aunque a pesar de los grandes avances, se siguieron presentando problemas disciplinarios al interior del aula de clase. Es de esperarse que las dinámicas culturales se repitan continuamente, estas corresponden a los modelos de comportamiento que el mismo contexto va grabando en el accionar humano.

Se identificó en estos recorridos que los estudiantes reproducían las mismas prácticas barriales: Uso de la violencia para alcanzar sus propósitos, incapacidad para establecer diálogos, lenguaje soez y jerarquías basadas específicamente en la fuerza o en su habilidad para jugar fútbol.

Como alternativa pedagógica, se hizo necesario propiciar desde el lenguaje propio el reconocimiento del espacio, cada persona arma su mundo, incluso en la escuela "el lenguaje se emplea para construir una interpretación aceptada por todos de los propósitos, los acontecimientos, las tareas y los contenidos curriculares de cada una de las actividades que se desarrollan en el aula" (Nuthall, 2000, p. 53). A partir de este se crean acuerdos, que se supone son necesarios para mejorar la convivencia y el aprendizaje. La estructuración del discurso se convierte entonces en protagonista del proceso educativo, y potencia un mejor escenario de enseñanza-aprendizaje, "tenemos que hacer del diálogo un instrumento esencial y asumir un aprendizaje permanente de la convivencia de en paz, respetándonos y enriqueciéndonos recíprocamente" (Sánchez, 2011, p.36). Solo a partir de esta práctica cultural se logrará un ambiente respetuoso y amable.

Los acuerdos son vitales en el proceso escolar, respetando estos, los estudiantes vivenciaron experiencias en grupo por fuera de la institución, esto posibilitó a educandos y docente conocerse de forma más sincera. La dinámica motivó que los niños identificaran personas, objetos, animales, espacios y hechos, pertenecientes a su propia vida. Cada estudiante quería ser protagonista de la narración en vivo de su entorno, se sentían seguros y dispuestos a comunicar, a establecer parámetros de reconocimiento de lo que cada uno vivía. Era necesario comprender que todos eran personajes distintos y que desde esa diferencia se debería estructurar una convivencia en la que todos estaban en igualdad de condiciones.

Los recorridos evidenciaron una riqueza expresiva e informal dispuesta por los estudiantes para mostrar su entorno socio-cultural. Las versiones distintas de todos aumentaron la complejidad de cada hecho narrado, fue muy importante respetar cada visión y construir desde el colectivo una mirada en ocasiones más profunda de lo discutido, ¿es probable que se tenga una alternativa de narración que informe a todos el mismo hecho? El periódico es una de estas, es un medio escrito que construye una historia para todos. La escritura es la herramienta que los estudiantes deben aprender para que sus ideas del mundo tengan una mayor claridad.

Se leían a diario noticias de la ciudad, ellos opinaban cuando hacían referencia a su sector, desafortunadamente, siempre fueron de carácter negativo, en algunos casos se tergiversaron los hechos en los medios. Después de dos meses de trabajo se identificó el deseo por escribir en los estudiantes, los recorridos fueron fundamentales para registrar en palabras escritas lo que ya se conocía.

Indagar en el conocimiento debe ser un ejercicio natural,

se trata de entrar en lo escrito a partir de la vida misma y de los textos en situación, y no a través de ejercicios y palabras fuera de contexto o de letras aisladas. Aun cuando los niños llegan a la escuela habiendo interrogado numerosos escritos, en sus casas o en otros lugares, el rol de la escuela para comprender el sentido de ellos es actuar de modo que los niños en edad escolar descubran más precisa y sistemáticamente el nuevo lenguaje a través de textos y todas las posibilidades que estos abren. (Jolibert \& Sraiki, 2009, p. 57) 
La escritura y la lectura fueron herramientas para comprender de otra forma el entorno.

En ese mismo orden de ideas, los estudiantes solicitaron la escritura de sustantivos, empezando en algunos casos por su propio nombre, la gran mayoría de las cosas que los inquietaban estaban presentes inicialmente en el hogar, mascotas y utensilios con las cuales tenían relación directa. La acción fue divertida, y se experimentaba un goce en el aprendizaje. Lo trascendental fue la motivación de conocer las palabras escritas y algunas frases con sentido, así fuera bajo dinámicas culturales ajenas a la moralidad que se determina en la escuela. Fue un proceso muy activo, no se realizaron evaluaciones escritas como los cánones de la institución lo estipulaban, es complejo reconocer que "los docentes se benefician de una autonomía aún mayor en los sistemas escolares que instituyen una evaluación continua” (Perrenoud, 2008, p.37). En este caso, la evaluación implica un proceso de corrección continuo y por supuesto un seguimiento individual de cada estudiante, reconociendo: intereses y resultados.

El ejercicio proyectual finalmente se contempló en el salón de clase y posibilitó constituir frases más elaboradas, en las que se identificaba un afán por abstraer vivencias en el ejercicio escrito. "El mejor ejercicio del cerebro es aquel que lo obliga a realizar operaciones de abstracción y razonamiento, es decir el ejercicio intelectual" (Campos, 2009, p. 18). Fue muy importante que desde los estudiantes mismos se interiorizaran las dinámicas de narración que le daban forma a cada entorno, vinculándolos con el reconocimiento del mismo a partir de lo que se estudiaba en el ambiente académico.

\section{RESULTADOS Y CONCLUSIONES}

El presente documento se determina como artículo de reflexión, concluyendo que el proceso de enseñanza aprendizaje debe estructurarse a través de un ambiente idóneo, coherente y contextualizado con los agentes discursivos implicados, además se reconoce que es una acción de respeto. Se sugiere que la relación profesor-estudiante, no debe ser una relación mediada por el temor o la desconfianza, por el contrario, debe estar respaldada en el deseo de enseñar por parte del educador y por el deseo de aprender del educando.

Por otra parte, la relación que se gesta en la academia implica principalmente la conformación de comunidad, cada agente inmiscuido debe recibir herramientas particulares para reconocer su espacio histórico, elemento trascendental para que el individuo comprenda lo que él es con relación a su entorno. Por tal motivo, las propuestas pedagógicas no se pueden determinar como camisas de fuerza o como moldes que van a perdurar de generación en generación; por el contrario, estas actúan como guía que en algún momento se pueden retomar o transformar, ubicando su contexto para aprovechar tanto sus dinámicas teóricas, como sus resultados pragmáticos, siempre aplicándolas a las necesidades particulares de aquellos procesos que encajen.

Lo que el ciudadano de hoy le pide al sistema educativo es que lo capacite para poder tener acceso a la multiplicidad de escrituras, de lenguajes y discursos en los que se producen las decisiones que lo afectan en el plano laboral, en el familiar, en el político y en el económico. (Martín, 2003, p. 27)

La capacidad para interpretar el mundo y construir desde este una postura, es el anhelado éxito. La palabra éxito conduce el ejercicio formativo hacia una culminación satisfactoria del proceso mismo.

Se especifican a continuación los resultados obtenidos al finalizar el proceso de observación, análisis y proposición de mejores alternativas de educación en el aula de clase: el grado primero de la escuela culminó su proceso escolar en el año 2004, el 95\% de los educandos aprendió a leer y escribir, además de establecer mejores relaciones humanas. Los ejercicios narrativos permitieron identificar dos casos muy complejos, por recomendación de especialistas, los niños fueron atendidos en sesiones psicológicas en la Facultad de Salud de la Universidad Santiago de Cali. Ambos casos correspondían a abusos 
sexuales, uno de ellos fue retirado de su casa por el Instituto de Bienestar Familiar. El otro continuó en terapias por un año más.

Es importante reconocer lo complejo que fue el proceso, pero se culminó de forma adecuada, aunque existieron casos que no cumplieron las expectativas presupuestadas: se distinguieron dos factores que incidieron en el resultado de cada metodología; la violencia intrafamiliar y la desnutrición, estas características fueron elementos de discordia entre los estudiantes, entre docente y estudiantes, entre padres y estudiantes, pero no entre padres y docentes, en esta última relación; los primeros avalaron el proceso y culpabilizaron a los niños reprobados, tras evaluaciones desfavorables. No se puede olvidar que: "la evaluación siempre es mucho más que una medida” (Perrenound, 2008, p. 73). Es una cuestión de jerarquías y dinámicas de poder bien establecidas en el aula.

El aula es una copia de la realidad, en el sentido que permite el traslado de vivencias a su interior. Muchos de estos factores están alejados del contexto escolar, pero inciden profundamente en las relaciones al interior del salón de clase. Las relaciones humanas son un tema obligado de asimilación y de reflexión en cada proceso pedagógico. Gloria Rincón comenta lo siguiente y se apoya en Lev Vygotsky para determinar que el sujeto es producto de las relaciones humanas que lo han rodeado durante su proceso de vida:

en el desarrollo cultural del niño toda función aparece dos veces: primero en el ámbito social $\mathrm{y}$, más tarde, en el individual; primero entre personas - interpsicológicas- y después en el interior del propio niño -intrapsicológica-. Esto puede aplicarse igualmente a la intención voluntaria, a la memoria lógica y a la formación de conceptos". (Rincón, 2003, p.67)

Lo anterior indica que los agentes discursivos construyen sus propias condiciones de convivencia, asumiendo cierto grado de dependencia y rol social, cada uno actúa bajo parámetros específicos aprehendidos a lo largo de su proceso de vida. La convivencia escolar es una de las principales preocupaciones de la educación infantil, se habla con regularidad de resolución de conflictos, de habilidades para la vida o de formación en valores. Cada perspectiva pedagógica se enmarca dentro de las relaciones humanas y su preocupación conlleva a mejorar la interacción entre los individuos pertenecientes a una comunidad. "Los estudiantes antes de llegar al aula presentan una historia particular, provienen de contextos sociales y culturales distintos, y asumen los aprendizajes a través de intereses que los ligan con un proyecto individual de vida" (Zambrano, 2001, p. 110). Para ello se deben tener en cuenta las condiciones de aprendizaje de cada uno, incluso las del docente, quien se convierte en el dinamizador del saber, en la evaluación.

Sería pertinente que docentes y estudiantes, en aras de asegurar un ejercicio educativo basado en la comunicación; se tuviera en cuenta que "La comunicación, en resumidas cuentas, es uno de los pocos fenómenos que cumple con los requisitos para ser sistema integrador, ya que un sistema social surge cuando la comunicación desarrolla nuevos tipos de comunicación, a partir de sí misma” (Monroy, 2001, p. 120). Ambos agentes discursivos están sujetos a una misma estructura comunicativa en la imposición de conocimiento; por lo menos quien educa debe conocer la procedencia de aquellos sujetos de quienes él es responsable, de esta forma, los agentes discursivos inmersos en el proceso, pueden dinamizar los distintos estados de saber a través de su relación enseñanza-aprendizaje. Las alternativas comunicativas que unen ambas generaciones (docentes-estudiantes) son en muchos casos incompatibles, por ello, es necesario la predisposición de las dos partes para lograr acceder a un vínculo comunicativo y evitar que los estudiantes caigan en la deserción escolar. "Casi se podría decir que uno de los factores que inciden en la repitencia o fracaso escolar es la distancia existente entre los lenguajes escolares y los lenguajes de los estudiantes". (Zambrano, 2001, p.120). La acción comunicativa se convierte en una alternativa de primera mano para estructurar una mejor relación entre los sujetos que componen la comunidad educativa y su entorno, el puente es el reconocimiento del saber. 
Se recomienda entonces, estructurar programas académicos más conscientes de los problemas reales de los estudiantes, lo que permitiría un ejercicio docente más sincero y coherente con las dinámicas que se proponen en la Misión y en la Visión institucional. De igual forma, estos lineamientos educativos deben construirse a partir de intereses básicos, como por ejemplo: el juego, el amor, la pertinencia, la frustración, el contexto y los sueños. Existe un interés por frustrar al niño y condicionarlo a comportarse, pensar e interpretar el mundo desde una visión más académica que humana. Los procesos formativos se inclinan más por convertir al estudiante en un contenedor que recibe conceptos imposibles de aplicar en su entorno. La escuela debe garantizar entonces: conocimiento, placer y aplicación del saber compartido.

El ejercicio de planeación educativa debe conocer las expectativas o necesidades de los estudiantes para lograr los mencionados objetivos

toda estrategia pedagógica, toda acción didáctica, supone el propósito de mediar, corregir y estimular la experiencia del encuentro entre sujetos que ejercen una serie de funciones sobre o con un contenido, [...] de suerte que quedan transformadas y enriquecidas dichas funciones y capacidades que en sentido general reconocemos como aprendizaje”. (Gimeno, 2010, p. 36)

Los sistemas educativos, en lugar de seducir a los estudiantes con temáticas contextualizadas o simplemente interesantes, terminan por obligarlos a la adaptación de un proceso adecuado.

La escuela entonces es el escenario preciso para que educadores y educandos se ofrezcan mutuamente un espacio para construir acuerdos de enseñanza - aprendizaje, teniendo en cuenta factores importantes como: la historicidad, la cotidianidad y la proyección de vida, motivando el deseo por unificar criterios de convivencia más allá de aquellas relaciones de poder que discriminan y golpean a los más débiles e inexpertos. Ahora el proceso se centra en posibilitar el diálogo entre agentes discursivos que participan con sus pretensiones de saber, creencias y valores al interior del escenario educativo.

La idea de motivar en el educando una disposición para que desde el reconocimiento de su contexto construya su proceso académico, va en contra vía del concepto formación, que manifiesta un ideal, la "formación comprende un sentido general de la mesura y de la distancia respecto asimismo, y en esta misma medida elevarse por encima de sí mismo hacia la generalidad" (Gadamer, 1997, p. 46). La observación arrojó otra mirada, aquellos pequeños construyen pactos, tienen intereses precisos, proyectan malestares familiares o barriales.

No se trata de construir una teoría sólida e irrefutable, por el contrario, el proceso claramente se muestra como una exploración que parte de las relaciones interpersonales y se dimensiona al entorno. Incluso la preocupación principal fue antes que enseñar a leer y a escribir, aprender a convivir. Elemento que fue duramente cuestionado al inicio del proceso, debido a que los padres de familia, al estar inmersos en la misma situación de violencia, no comprendían el afán expuesto con relación a trabajar en las relaciones sociales de sus hijos. Fue un ejercicio de concientización lento, lleno de expectativas y frustraciones, pero que valió la pena.

Mejorar las relaciones sociales fue siempre un interés de la Institución, aunque se le otorgaba al castigo (físico y psicológico) una opción de corrección. De forma paulatina este ejercicio del poder fue desapareciendo del aula, sin mayores traumatismos, "es feo ser digno de castigo, pero poco glorioso castigar” (Foucault, 2005, p. 17).

\section{REFERENCIAS BIBLIOGRÁFICAS}

Beltrán, D. (2005). Comunicación dialógica, democracia y discurso pedagógico, Bogota: Universidad Distrital Francisco José de Caldas Beuys, J. (1995). Cada hombre, un artista, Madrid: Visor.

Campos, M. (2009). La lectura piedra angular del futuro en: el poder d ella lectura, Mexico D.F.: Trillas. 
Cano, F. (2006). Coordinador Javier Murillo, factores de logro cognitivo en la escuela primaria colombiana. Estudio realizado sobre una muestra de planteles - grados $3^{\circ}, 5^{\circ}$ - (19931994) en: estudios sobre eficacia escolar en Iberoamérica, Bogotá: Convenio Andrés Bello

Cárdenas, A. (2000). El docente como protagonista del cambio educativo en: el maestro protagonista del cambio educativo, Bogotá: Cooperativa Editorial Magisterio, Convenio Andrés Bello y Corporación Tercer Milenio.

Concha, C. (2006). Coordinador Javier Murillo, estudio de 32 escuelas de alta vulnerabilidad socioeconómica y altos resultados de aprendizaje en: estudios sobre eficacia escolar en Iberoamérica, Bogotá: Convenio Andrés Bello

Foucault, M. (2005). Trigésimo cuarta edición, el cuerpo de los condenados en: vigilar y castigar, México D.F.: Siglo XXI editores

Gadamer, H. (1997). Verdad y método, Salamanca: Sígueme S.A.

Gimeno, J. (2010a). El sentido del currículo en la enseñanza obligatoria en: saberes e incertidumbres del currículo, Madrid: Morata.

Gimeno, J. (2010b). ¿qué significa el currículo? en: saberes e incertidumbres del currículo, Madrid: Morata.

Iglesias, Leonardo (2007). Conducta y normas en: la cultura contemporánea y sus valores, Barcelona: Anthropos

Jolibert, J. \& Sraiki, Ch. (2009). Qué es aprender a leer/escribir en la escuela en: Niños que construyen su poder de leer y escribir, Buenos Aires: Manantial

Martín, J. (2003). Coordinadora Roxana Morduchowicz, retos culturales de la comunicación a la educación, elementos de una reflexión que está por comenzar en: Comunicación, medios y educación, Barcelona: Octaedro S.L.
Meirieu, P. (2002). ¿Podemos aprender? en: Aprender, sí. Pero ¿Cómo?, Barcelona: Octaedro, S.L.

Meirieu, P. (2004). La escuela no es sólo un servicio, también es una institución en: en la Escuela hoy, Barcelona: Octaedro, S.L.

Monroy, B. (2001). Los ensayos de los maestros en: quién dijo que es fácil escribir, Bogotá: Universidad Externado de Colombia

Murcia, J. (1991). Proceso pedagógico y evaluación Bogotá D.C. Bogotá: Siglo XXI

Nuthall, G. (2000). Compiladores Bruce J. Biddle et al., el razonamiento y el aprendizaje del alumno en el aula en: la enseñanza de los profesores II la enseñanza y sus contextos, Barcelona: Paídos.

Ortiz, A. (2011). Hacia una nueva clasificación de los modelos pedagógicos: el pensamiento configuracional como paradigma científico y educativo del siglo XXI, Revista Praxis, Volumen 7, Santa Marta: Universidad del Magdalena.

Perrenoud, P. (2008). La evaluación en el principio de ella excelencia y del éxito escolares en: la evaluación de los alumnos, de la producción de la excelencia a la regulación de los aprendizajes. Buenos Aires: Colihue.

Rincón, G. (2003). Entre - Textos, la comprensión de textos escritos en la educación primaria, Cali: cátedra UNESCO - Universidad del Valle

Sanchez, I. (2011). Enfoques y modelos de educación intercultural, Revista Praxis, Volumen 7, Santa Marta: Universidad del Magdalena

Vásquez, G. (2009). Coordinadores José Vicente Peña y Carmen Fernández, la escuela en crisis, Barcelona: Octaedro

Zambrano, A. (2001). Pedagogía, educabilidad y formación de docentes, capitulo 1, Cali: Nueva Biblioteca Pedagógica. 